\title{
Study On The Target Positioning For An Omni-Directional 3 DOF Mobile Manipulator Based On Machine Vision
}

\author{
Jiwu Wang \\ School of Mechanical, Electronic and Control Engineering, Beijing Jiaotong University \\ Beijing 100044, China ${ }^{1}$ \\ Yao Du ${ }^{1}$, Wensheng Xu \\ School of Mechanical, Electronic and Control Engineering, Beijing Jiaotong University \\ Beijing 100044, Chinal \\ Masanori Sugisaka \\ Alife Robotics Corporation Ltd, Japan and Open University, United Kingdom ${ }^{2}$ \\ E-mail:15121244@bjtu.edu.cn ${ }^{1}$;ms@alife-robotics.co.jp ${ }^{2}$ \\ www.bjtu.edu.cn
}

\begin{abstract}
The omni-directional mobile robot with multi DOF, because the operation posture and operation accuracy of the manipulator can be better controlled in a narrow or crowded workplace compared with the general manipulator, is getting more interested in practical applications. The present problem is to improve its flexibility for operating multiple different targets. Target recognition with image processing is an effective solution. Based on the image processing, the position and posture of the target can be determined. Then the signal will be sent to the arm control system. In this paper, the illumination conditions, distortion, etc. are studied in the target recognition. The target position with image processing, is verified with real coordinates. The experiments show target recognition with image processing can effectively improve the flexibility of our robot.
\end{abstract}

Keywords: mobile robot; machine vision; 3 DOF manipulator; Omni-direction.

\section{Introduction}

Today, the degree of automation of factories and warehouses becomes increasingly higher and higher. ${ }^{1}$ However it is difficult to expand manipulator's functions because its fixed base. This article describes an Omni-directional 3 DOF mobile manipulator, which can increase flexibility of the manipulator and greatly increase its function.

Firstly, through the analysis of the 3 DOF manipulator, established the manipulator kinematics model by the
D-H method, and on the basis of this model, derived solving method to the manipulator kinematics equation of the manipulator and the inverse kinematics problem, the analytical solution of problem of manipulator inverse kinematics.

Then the analytical solutions of the forward kinematics equations and inverse solutions are verified and analyzed.

The experimental results prove the effectiveness of the method and the method of the target location. 


\section{System Overview}

In order to realize the automatic control of the robot, the robot control system plays an indispensable role. In this paper, the robot uses four Mecanum wheels to realize the omnidirectional movement of the robot platform. Robot control system is divided into four parts, they are IPC, the main, chassis drives and mechanical arm drive plate, as shown in Figure 1.

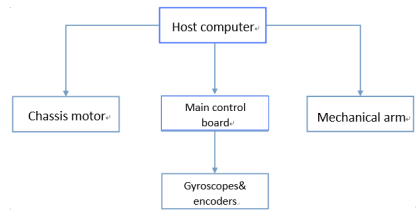

Fig.1. System Overview

IPC runs the Windows operating system, through the serial port and the main control board to achieve communication between the main control board and the two driver boards through the CAN bus communication.

\subsection{Design of Omnidirectional Mobile Chassis Based on Mecanum Wheel}

In this paper, four Mecanum wheels are installed to realize the omni-directional movement of the platform, as shown in Figure 2. ${ }^{2}$

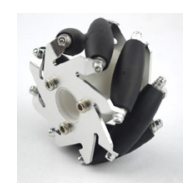

Fig.2. Mecanum wheel

From Figure 3 you can see the four Mecanum wheels combined installation situation. Each wheel is driven by a DC brush motor alone, with both left-handed and right-handed rotations.

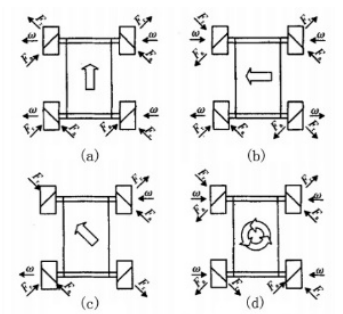

Fig.3.Analysis of chassis movement
Figure 4 shows the Mecanum wheel four-wheel robot chassis model coordinate system. The coordinate system $O_{i} X_{i} Y_{i}(i=1,2,3,4)$ is established with the center of each wheel position as the origin, and the reference coordinate system $O X Y$ is established with the center of the robot as the origin.

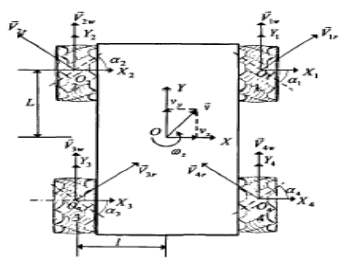

Fig.4. Coordinate modeling of omnidirectional mobile robot

As shown in Figure4, the robot body length is $2 \mathrm{~L}$, the body width is 2 , the speed of the robot chassis is $\vec{V}=$ $\left(V_{x}, V_{y}\right)$, the angular velocity of the robot around the Z-axis is $\omega_{z}$, the linear velocity of the wheel is $\vec{V}_{i w}$, there $\vec{V}_{i w}=\omega_{i} R$. (Where $\omega_{i}$ is the angular velocity of each wheel and $\mathrm{R}$ is the radius of the wheel). The roller and the ground contact when the line speed $\vec{V}_{i r}$. Suppose that the speed of each wheel is $\vec{V}_{i}=\left(V_{i x}, V_{i y}\right)$. Under $O_{i} X_{i} Y_{i}(i=1,2,3,4)$, can be decomposed along $X_{i}$ and $Y_{i}$, as $\vec{V}_{i w}$ and $\vec{V}_{i r}$, and $V_{i x}$ can be expressed as $-V_{i r} \sin \alpha_{i}, V_{i y}$ is the sum of $\vec{V}_{i w}$ and $\vec{V}_{i r} \cos \alpha_{i}$. Which satisfy the relation:

$V_{1 x}=-V_{1 r} \sin \alpha_{1}, \quad V_{1 \mathrm{y}}=-V_{1 w}+\mathrm{V}_{1 r} \cos \alpha_{1}$

$V_{2 x}=-V_{2 r} \sin \alpha_{2}, \quad V_{2 y}=-V_{2 w}+V_{2 r} \cos \alpha_{2}$

$V_{3 x}=-V_{3 r} \sin \alpha_{3}, \quad V_{3 y}=-V_{3 w}+V_{3 r} \cos \alpha_{3}$

$V_{4 x}=-V_{4 r} \sin \alpha_{4}, \quad V_{4 \mathrm{y}}=-V_{4 w}+\mathrm{V}_{4 r} \cos \alpha_{4}$

In the formula $\alpha i$-i round roller axis and the hub axis angle $(i=1,2,3,4)$.

Overall, the speed of each wheel $\vec{V} i$ is the speed of the robot and the relative speed of the wheel center $\mathrm{O}$ speed vector, that is, $\vec{V}=\vec{v}_{i}+\vec{\omega}_{\mathrm{z}} * \overrightarrow{00}_{\mathrm{l}}$, consider vector directionality, along the coordinates and $X_{i} Y_{i}$ Coordinate decomposition formula can be obtained:

$V_{1 x}=V_{x}-\mathrm{L} \omega_{z}, \quad V_{1 y}=V_{y}+l \omega_{z}$

$V_{2 x}=V_{x}-\mathrm{L} \omega_{z}, \quad V_{2 y}=V_{y}-1 \omega_{z}$

$V_{3 x}=-V_{x}+\mathrm{L} \omega_{z}, \quad V_{3 y}=V_{y}-1 \omega_{z}$

$V_{4 x}=V_{x}+\mathrm{L} \omega_{z}, \quad V 4_{y}=V_{y}+{ }_{l} \omega_{z}$

Taking into account the pipe speed for the non-controlled volume, to eliminate, that is derived omni-directional robot inverse kinematics equation is:

$W_{l}=\frac{1}{R}\left[-V_{x} \cot \alpha+V_{y}+(l+L \cot \alpha) \omega_{z}\right]$ 
$W_{2}=\frac{1}{\mathrm{R}}\left[V_{x} \cot \alpha+V_{y}-(l+L \cot \alpha) \omega_{z}\right]$

$W_{3}=\frac{1}{R}\left[-V_{x} \cot \alpha+V_{y}-(l+L \cot \alpha) \omega_{z}\right]$

$W_{4}=\frac{1}{R}\left[-V_{x} \cot \alpha+V_{y}+(l+L \cot \alpha) \omega_{z}\right]$

From the above equation, the control of the whole robot can be realized.

\subsection{Design of Multi - DOF Gripping Manipulator}

The design of the robot arm a total of three degrees of freedom, the direction of the $\mathrm{X}$-axis rail, two connecting rods were $\mathrm{Y}, \mathrm{Z}$ axis.

\subsubsection{Motion Control of Manipulator 's Hand}

\section{Linear Interpolation}

Calculation shows that the position of the robot joint position and the relationship between the hand space:

$$
\left\{\begin{array}{c}
\theta_{2}=a \cos \frac{p_{y}^{2}+p_{z}^{2}-l_{1}^{2}-l_{2}^{2}}{2 l_{1} l_{2}} \\
\theta_{1}=a \cos \frac{p_{y}\left(l_{1}+l_{2} \cos \theta_{2}\right)-p_{z} l_{2} \sin \theta_{2}}{\left(l_{1}+l_{2} \cos \theta_{2}\right)^{2}+l_{2}^{2} \sin ^{2} \theta_{2}}
\end{array}\right.
$$

For the robot hand space position, the line is interpolated as it moves from $\left(y_{1}, z_{1}\right)$ to $\left(y_{2}, z_{2}\right)$ in a straight line.

Because of simple interpolation from the $\mathrm{x}$-axis or $y$-axis, there is a great deal of non-uniformity.

Assuming the slope of the line segment is $\mathrm{k}$, when $y_{1} \neq y_{2}, k=\left(z_{2}-z_{1}\right) /\left(y_{2}-y_{1}\right)$, the straight line inclination angle is:

$$
\alpha=\operatorname{arctank}
$$

Assuming that $\mathrm{d}$ is the distance from any point $(y, z)$ to $\left(y_{1}, z_{1}\right)$ on this line segment, the point corresponding to $\mathrm{d}(y, z)$ is

$$
\left\{\begin{array}{l}
y=y_{1}+d \cos \alpha \\
z=z_{1}+d \cos \alpha
\end{array}\right.
$$

Where $\mathrm{d}$ is in the range of $\left[0, \sqrt{\left(x_{2}-x_{1}\right)^{2}+\left(y_{2}-y_{1}\right)^{2}}\right]$. When $\mathrm{d}$ is evenly changed in this interval, the movement of the hand $(y, z)$ obtained by the above-described equation is substituted into the joint position expression so as to be able to realize a straight line movement of the hand

\subsubsection{Robot manipulator arm speed control}

It can be seen that the relationship between the spatial position of the robot hand and the joint variable is

$$
\left\{\begin{array}{l}
p_{y}=l_{2} c_{1} c_{2}-l_{2} s_{1} s_{2}+l_{1} c_{1} \\
p_{z}=l_{2} c_{2} s_{1}-l_{2} c_{1} s_{2}+l_{1} s_{1}
\end{array}\right.
$$

Derivative of the above formula can be obtained

$$
\left\{\begin{array}{c}
d p_{y}=\frac{\partial y}{\partial \theta_{1}} d \theta_{1}+\frac{\partial y}{\partial \theta_{2}} d \theta_{2}=\left(-l_{2} s_{12}-l_{1} s_{1}\right) d \theta_{1}+\left(-l_{2} s_{12}\right) d \theta_{2} \\
d p_{z}=\frac{\partial z}{\partial \theta_{1}} d \theta_{1}+\frac{\partial z}{\partial \theta_{2}} d \theta_{2}=\left(l_{2} s_{12}-l_{1} c_{1}\right) d \theta_{1}+\left(l_{2} c_{12}\right) d \theta_{2}
\end{array}\right.
$$

Written in matrix form

$$
\left[\begin{array}{l}
d P_{y} \\
d P_{z}
\end{array}\right]=\left[\begin{array}{cc}
-l_{2} s_{12}-l_{1} s_{1} & l_{2} c_{1} c_{2} \\
l_{2} c_{12}+l_{1} c_{1} & l_{2} c_{12}
\end{array}\right]\left[\begin{array}{l}
d \theta_{1} \\
d \theta_{2}
\end{array}\right]
$$

Is known from the definition of the Jacobian matrix:

$$
\dot{p}=J \dot{q}
$$

The Jacobian matrix of the manipulator can be obtained

$$
J=\left[\begin{array}{cc}
-l_{2} s_{12}-l_{1} s_{1} & l_{2} c_{1} c_{2} \\
l_{2} c_{12}+l_{1} c_{1} & l_{2} c_{12}
\end{array}\right]
$$

Jacques array is mainly used to describe the joint displacement and space pose differential relationship, the relationship between the spatial velocity of the robot hand and the angular velocity of the joint is given

$$
v=J \dot{\theta}
$$

Conversely, the speed of movement of each joint can be determined from the desired hand speed $\dot{\theta}=J^{l} v$. According to calculations:

$$
\mathrm{J}^{-1}=\left[\begin{array}{cc}
-\frac{c_{12}}{l_{1} c_{12} s_{1}}-l_{1} s_{12} c_{1} & -\frac{s_{12}}{l_{1} c_{12} s_{12}}-l_{1} s_{12} c_{1} \\
\frac{l_{2} c_{12}+l_{1} c_{1}}{l_{1} l_{2} c_{12} s_{1}}-l_{1} l_{2} s_{12} c_{1} & \frac{l_{2} s_{12}+l_{1} s_{1}}{l_{1} l_{2} c_{12} s_{1}}-l_{1} l_{2} s_{12} c_{1}
\end{array}\right]
$$

\subsection{Design of Image Processing.}

Template matching is to find the target image in the image. ${ }^{3}$ The principle is to measure the similarity between two images by some similarity criteria.

\subsubsection{Template Matching Based on Gray Value}

When the template slides in the image, do similarity matching. The simplest measure of similarity is to calculate the sum of the absolute values of the differences between the template and the image or the sum of squares of all the differences (SAD and SSD).

$\operatorname{sad}(r, c)=\frac{1}{n} \sum_{(u, v) \in T}|t(u, v)-f(r+u, c+v)|$
$\operatorname{ssd}(r, c)=\frac{1}{n} \sum_{(u, v) \in T} x|t(u, v)-f(r+u, c+v)|^{2}$ 
$(r+u, c+v)$ denotes the gray value of the row $\mathrm{r}+\mathrm{u}$ column $\mathrm{c}+\mathrm{v}$ of the image to be measured, and $t(r, c)$ denotes the gray value of the template image $T$ row $r$ column c. ${ }^{4}$ The difference between the values after the change and their mean values will be smaller regardless of whether all the gray values become larger or smaller and then become zero mean and unit variance by dividing the variance so that the image is less sensitive to changes in the brightness level, and has strong robustness.

$n c c(r, c)=\frac{1}{n} \sum_{(u, v) \in T} \frac{t(u, v)-m_{c}}{\sqrt{s_{t}^{2}}} \cdot \frac{f(r+u, c+v)-m_{f}(r, c)}{\sqrt{s_{f}^{2}(r, c)}}$

$m_{c}$ is the average gray value of the template, $s_{t}{ }^{2}$ is the variance of all the gray values of the image to be measured, $(r$,$) is the average gray value of the image to$ be measured.

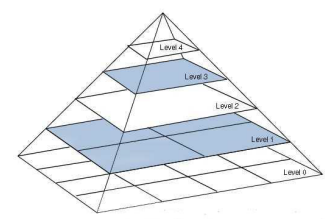

Fig.5.A schematic diagram of the image pyramid

As shown in figure 5, in the pyramid-based search strategy: from the top to the bottom of the search, in the high-level image search to track the template instance to the bottom of the image pyramid, then in a small area to match, in the next search region to locate the results of the matching of a small area the similarity degree is calculated, segmented by the threshold and the local extrema is extracted.

\subsubsection{Realization of crawl}

In order to achieve the robot automatic items crawl, we must get the actual location of items in the world coordinate system.

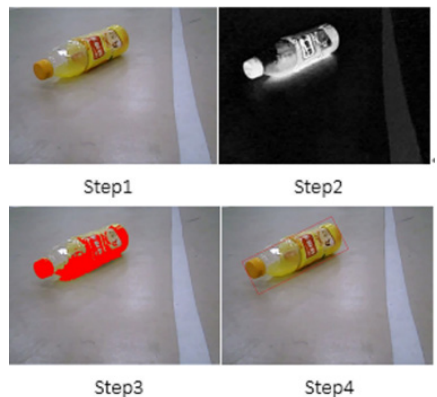

Fig.6. Steps to find items
As shown in figure 6. First, the original image distortion correction and smoothing noise; then extract the image of the saturation $\mathrm{S}$ component from the image HSV space, we can clearly see that saturation component of the object we want to crawl is relatively high; The third step is to get the image region with high saturation through the dynamic threshold technique. The final step is to display the obtained area and get the orientation information of the area and the orientation information of the bottle.

\section{Conclusion}

This paper introduce an Omni-directional 3 DOF mobile manipulator based on machine vision. However, in real-world experiments, the robot was controlled to grasp the bottle. Our experiments have shown that the desired relative position and orientation between the camera and the object have been kept within errors of few centimeters. The next step is to further improve the image processing method to make the image processing faster, so that the robot more accurate understanding of the surrounding environment. Adding some sensors, such as lidar, gives the robot a path planning and obstacle avoidance function. Increase the chassis suspension system to adapt to a richer terrain.

\section{References}

1. Wang J, Zhang $\mathrm{X}$ and Zhang W, Study on the Improvement of Flexibility for an Industrial Robot based on Machine Vision, J. Journal of Robotics, Networking \& Artificial Life, 2014, 1(1):45-48.

2. Du B, Zhao J and Song C, Dexterity Analysis for Omni-directional Wheeled Mobile Manipulator Based on Double Quaternion, J. Chinese Journal of Mechanical Engineering,2013,03:585-593.

3. Wang J, Zhang $X$ and Dou H, Study on the Target Recognition and Location Technology of Industrial Sorting Robot based on Machine Vision, J. Journal of Robotics, Networking \& Artificial Life, 2015, 2(2):108-110.

4. Wang J, Dou H and Zheng S, Study on the Target Recognition based on Machine Vision for Industrial Sorting Robot, J. Journal of Robotics, Networking \& Artificial Life, 2015, 2(2):100-102. 